\title{
Stimulus devaluation and extinction of chain schedule performance
}

\author{
BEN A. WILLIAMS \\ University of Califormia, San Diego, La Jolla, California \\ BERTRAM O. PLOOG \\ Hunter College, New York, New York \\ and \\ MATTHEW C. BELL \\ University of California, San Diego, La Jolla, California
}

\begin{abstract}
Pigeons were trained on a two-component multiple schedule in which each separate component consisted of a three-link chain schedule. After initial baseline training, the stimuli correlated with the terminal links of each chain were presented in a successive discrimination, with one stimulus continuing to be associated with reinforcement while responses to the alternative stimulus were extinguished. Subjects were then returned to the original chain schedule, but with extinction in effect in both components of the multiple schedule. In two separate experiments, extinction of initial-link responding was not affected by which terminal link had been extinguished during the separate discrimination training, indicating that devaluation of the terminal link was not transmitted directly to the initial link of the chain. There was also no effect of the devaluation procedure during the first session of testing on responding in the middle link of the chain, but an effect did develop with continued extinction of the entire chain when the terminal components were presented during extinction. When the terminal components were omitted, however, the latter effect did not occur. Also, when the terminal link was omitted, extinction occurred more rapidly in the middle component than in the initial component, indicating a backward pattern of extinction.
\end{abstract}

A major development in the study of operant behavior has been the appreciation that subjects are sensitive to the specific nature of the reinforcer contingent on the operant response. In a variety of studies (e.g., Adams \& Dickinson, 1981; Colwill \& Rescorla, 1986), animals have been trained with an operant contingency, and then the reinforcer contingent on the response has been devalued (typically by taste aversion conditioning) in the absence of availability of the response. When the response is then tested without further exposure to reinforcement, its rate is substantially and immediately reduced, relative to control conditions where the reinforcer contingent on a second response has not been devalued. Such results have sponsored the view that "response strength" does not adequately characterize the determinants of the level of operant behavior, and that some notion of direct response-reinforcer associations must be invoked instead (e.g., Dickinson, 1980).

This research was supported by grants from the National Science Foundation. Reprint requests should be addressed to the first author, Department of Psychology, University of California, La Jolla, CA 92093-0109 (e-mail: bawilliams@ucsd.edu).

-Accepted by previous editor, Vincent M. LoLordo
While the results noted above indicate that operant responding may be sensitive to the current value of its contingent reinforcer in simple training procedures, the role of such effects in more complex situations remains to be determined. Consider a chain schedule of reinforcement. Traditional analyses (e.g., Keller \& Schoenfeld, 1950) of chain schedule performance have assumed that the reinforcer maintaining such behavior is not the food at the end of the chain, but rather access to the stimulus for the next link in the chain. Thus, the stimulus for the terminal link acquires its value because of its direct association with food, while the middle-link stimulus (of a three-link chain) acquires its value by association with the terminal-link stimulus via the process of higher order conditioning. Responding during the initial-link stimulus then occurs because of the contingency between the response and the onset of the middle-link stimulus. The graded response rates across the different links of the chain are explained by each additional link having diminishingly little value because each additional step of higher order conditioning attenuates the level of value transmission.

This traditional analysis of chain-schedule performance has been challenged by Staddon (1983), who has argued that the controlling variable for behavior early in the chain is the temporal distance to the end of the chain 
signaled by the individual stimuli composing the chain (see Gollub, 1977, for a discussion of related evidence). This implies that the initial-link stimuli are associated with the primary reinforcer at the end of the chain in the same qualitative manner as are the terminal-link stimuli, although with a weaker ability to evoke responding because they cue a greater temporal distance to reinforcement.

One approach to understanding the dynamics of chainschedule performance is to examine the pattern of extinction. According to Staddon's (1983) account, the associative properties of the successive links of the chain differ only quantitatively, in terms of the different times to reinforcements predicted by each successive stimulus. Thus, the presumed implication for extinction (Staddon himself does not address the pattern of extinction) is that the decline in rate controlled by each stimulus should be similar across all links of the chain. Alternatively, when combined with the concept of "resistance to change" proposed by Nevin, Mandell, and Yarensky (1981), which implies that after a change in contingencies weaker behavior should change more rapidly than strong behavior, extinction should be more rapid in the early than in the later links of the chain.

In contrast, the traditional account of chain-schedule performance seems to imply a backward pattern of extinction. As the association of the terminal-link stimulus with food is extinguished, its loss of value is transmitted back to the middle link, which then itself must lose value before extinction of initial-link responding can occur. While the rates at which such backward transmission of value changes are unknown, the idea of backward extinction implies that extinction of responding should be evident in the middle link before becoming evident in the initial link, because the latter depends on the loss of value of both of the following components, while the former requires only the loss of value of the terminal link.

Whether the backward pattern of extinction actually does occur is not clearly answered by existing data. Early authoritative accounts (Keller \& Schoenfeld, 1950 , p. 202; Skinner, 1938, pp. 102-108) reported that all elements of the chain prior to the point of extinction declined together but without clear supporting evidence. Subsequent data on the pattern of extinction in chain schedules are surprisingly sparse, but a backward pattern of extinction was observed by Fantino (1965) using pigeons in a concurrent chains procedure. However, these findings are compromised by the fact that the different links of the chain were associated with different schedules of reinforcement (variable intervals in the initial links, fixed-ratio schedules in the terminal links), so that the different rates of extinction may have been due to other factors.

The central premise of the traditional account of chain-schedule performance is that the value of the stimuli correlated with the early links of the chain is mediated by higher order conditioning. This implies that extinction of the terminal component of the chain schedule, outside of the chain sequence, should be ef- fective in reducing behavior in earlier links of the chains, just as the removal of food at the end of the entire chain. It is unclear, however, whether such effects should be immediate or gradual. An immediate reduction in responding for early-link behavior, after extinction of the terminal link, should be expected from studies of second-order conditioning with the autoshaping preparation. When one stimulus, $\mathrm{S} 1$, is paired with the reinforcer, and a second stimulus, $\mathrm{S} 2$, is then paired with $\mathrm{S} 1$ in the absence of the reinforcer, thus producing secondorder conditioning to $\mathrm{S} 2$, separate extinction of $\mathrm{S} 1$ has resulted in an immediate reduction of responding to $\mathrm{S} 2$ (Nairne \& Rescorla, 1981; Rashotte, Griffin, \& Sisk, 1977; Rashotte, Marshall, \& O'Connell, 1981; Rescorla, 1979). This immediate sensitivity of $S 2$ responding to the value of S1, without further S2-S1 pairings, has been interpreted as showing that $\mathbf{S} 2$ is associated with the sensory representation of $\mathrm{S} 1$, so that the value possessed by $\mathrm{S} 1$, however modulated, is transferred immediately to $\mathrm{S} 2$. Similar dynamics might be expected in chain schedules involving keypecking in pigeons, given the similarity to the autoshaping procedure. On the other hand, not all studies of second-order conditioning have produced the pattern of results just described (e.g., Holland \& Rescorla, 1975), and it remains unclear what the critical determinants are of the effects of extinction of S1. However, even if the effects of the extinction of the terminal link of a chain schedule are not immediate, performance in the links of the chain preceding that terminal link should still be attenuated with continued nonreinforced presentations of the chain relative to a similar chain in which no separate extinction of the terminal link has occurred.

The present study provides an empirical assessment of the pattern of extinction in three-link chain schedules. In addition, it examines the effect of separate extinction of the terminal-link stimulus on responding in the preceding links of the schedule, in a manner analogous to that used by Rashotte et al. (1977). Pigeons were trained on a multiple schedule with each component consisting of a three-link chain. Then, outside the chain sequences, the stimuli correlated with the terminal links of the two chains were used in discrimination training, such that one stimulus was further associated with reinforcement while the other was correlated with extinction. After this discrimination had been learned, subjects were returned to the original multiple schedule but with food removed from the terminal links for both components. At issue was (1) whether the separate extinction training would be immediately evident in the early links of the chain associated with extinction, and (2) the pattern of extinction for the different links of the chain.

\section{EXPERIMENT 1}

\section{Method}

\section{Subjects}

Twelve White Carneaux pigeons with various experimental histories were maintained at $80 \%$ of their free-feeding body weights 
by additional feeding, when necessary, after the end of the experimental sessions. Subjects were housed individually in stainless steel cages in a colony room with an 18-h-on, 6-h-off light cycle. Water and grit were available at all times.

\section{Apparatus}

Four identical three-key pigeon chambers were used. They were cylindrical in shape, $36 \mathrm{~cm}$ high and $33 \mathrm{~cm}$ in diameter. The response keys were located $24 \mathrm{~cm}$ above the mesh floor and $7 \mathrm{~cm}$ apart, center to center. A force of approximately $0.10 \mathrm{~N}$ was required to operate the response keys and produce an audible feedback click. A white houselight was located $8 \mathrm{~cm}$ above the center key and was illuminated except during reinforcer presentations. The food hopper opening ( $5 \mathrm{~cm}$ high and $5 \mathrm{~cm}$ wide) was located $16 \mathrm{~cm}$ below the center key. Reinforcement was delivered via a solenoid-operated food hopper and consisted of 3-sec access to milo. During reinforcer presentations, the houselight and response keys became dark and the hopper opening was illuminated. A ventilating fan, which also provided masking noise, was located in the lid of the chamber. An IBM-compatible 286 computer, located in an adjacent room, controlled and recorded the experimental events in all chambers.

\section{Procedure}

Pretraining. Autoshaping was provided during the first session with respect to all of the stimuli to be used in the chain schedules. During an intertrial interval (ITI) of $90 \mathrm{sec}$, the houselight, but no keylight, was presented. At the termination of the ITI, the left key was lit with one of six stimuli: a blue, red, green, white, or white horizontal or vertical line. The line stimuli consisted of white lines ( $2 \mathrm{~mm}$ in width and $2 \mathrm{~cm}$ in length) on a dark background. Each stimulus had an equal probability of presentation. A trial was terminated either by a peck to the lit key or after $5 \mathrm{sec}$ if no response had occurred. In either case, termination of a trial resulted in food presentation and the onset of the next ITI. A session continued until 50 reinforcements had been presented. Autoshaping sessions were continued until reliable pecking occurred to all six stimuli (usually only one session was required).

Baseline training. The experimental design consisted of two components of a multiple schedule, presented quasi-randomly, with each component consisting of a chain schedule with three successive links, with different stimuli for each link. All six stimuli were presented on the left key. Each chain had an equal probability of presentation, with the restriction that no more than three presentations of each could occur successively. Each chain was presented 16 times during each session. Advance from the initial to the middle and from the middle to the terminal link of each chain was response-dependent, according to a variable-interval (VI) 30-see schedule. Food was delivered at the end of the terminal links of both chains, also according to VI 30-sec schedules. Baseline training was in effect for a total of 25 sessions.
Devaluation training. In this phase, all subjects were exposed only to the stimuli of the terminal links of the chains used in baseline training. These stimuli were presented randomly for a total of 16 times for each stimulus. Responses to one of the two stimuli resulted in reinforcement according to the VI 30-sec schedule of baseline training while responses to the other stimulus were extinguished. During the extinction (EXT) trials, presentation of the stimulus terminated independently of responding according to the same VI 30-sec schedule that scheduled food during the alternative stimulus. Which stimulus was associated with EXT versus the VI 30 -sec schedule was counterbalanced across subjects. Devaluation training continued for 20 sessions for subjects in Group 1 and for 10 sessions for subjects in Groups 2 and 3 (see below).

Test. For the next five sessions, all birds were returned to the baseline condition with the modification that no food was delivered at any time. Termination of the terminal link of both chains was independent of responding, governed by a variable-time (VT) 30 -sec schedule. Advancement within the chain from the initial to middle and from the middle to terminal links continued to be response dependent according to the same VI $30-\mathrm{sec}$ schedule as used during baseline training.

Reversal of contingencies. After the initial test, subjects were returned to the baseline for 25 sessions of retraining with the reinforcement contingencies intact. Devaluation training was then conducted again, but with the stimuli correlated with reinforcement versus extinction reversed from their original assignment. A second test with extinction in effect, identical in all respects to that described above, was again conducted.

Group assignments. The experiment was conducted in three replications with four subjects in each group. The primary difference between groups was the assignment of stimuli to schedule components, as shown in Table 1. For Group 1, the stimuli for the initial, middle, and terminal links of Chain 1 were red, horizontal line, and blue, respectively, while those for Chain 2 were green, vertical line, and white, respectively. For Group 2, the corresponding stimulus assignments were horizontal line, red, and blue for Chain 1 and vertical line, green, and white for Chain 2. For Group 3, the stimulus assignments were counterbalanced over subjects. For 2 subjects, the stimuli were red, green, and horizontal line for Chain 1 and white, blue, and vertical line for Chain 2 . For the remaining 2 subjects, the stimuli were green, red, and horizontal line for Chain 1 and blue, white, and vertical line for Chain 2 .

In addition to the stimulus assignments, Groups 2 and 3 differed from Group 1 in terms of a 30-sec ITI separating the end of the terminal links and the beginning of the initial link. During this ITI, all keylights were extinguished and pecking had no effect. For Group 1, the end of the terminal link was followed immediately by access to the next initial link. The ITI was added because subjects in Group 1 were very slow to extinguish during the devaluation sessions, so that 20 sessions of discrimination training were re-

Table 1

Stimuli Correlated With Each Link of Each Chain for the Three Groups of Subjects

\begin{tabular}{ccclll}
\hline Group & ITI & Chain & Initial Link & Middle Link & Terminal Link \\
\hline 1 & 0 sec & 1 & Red & Horizontal line & Blue \\
& & 2 & Green & Vertical line & White \\
2 & $30 \mathrm{sec}$ & 1 & Horizontal line & Red & Blue \\
& & 2 & Vertical line & Green & White \\
3 & $30 \mathrm{sec}$ & 1 & Red/Green & Green/Red & Horizontal line \\
& & 2 & Blue/White & White/Blue & Vertical line \\
\hline
\end{tabular}

Note-Group 1 had no ITI between the successive chain presentations, while Groups 2 and 3 received a $30-\sec$ ITI 
quired. For Groups 2 and 3, only 10 sessions of discrimination training occurred during each devaluation phase.

\section{Results}

Figure 1 shows the mean response rates in each link of the chain schedule during the last five sessions of baseline training and averaged across all three groups with different stimulus assignments. Data are shown separately for the initially presented (top) and reversal (bottom) phases of the experiment. Also shown separately are the chains that were later to be treated differentially with respect to the continued reinforcement (RF) or extinction (EXT) of their terminal links (open bars refer to the chain that later received extinction; filled bars refer to the chain that later received continued reinforcement). Because subjects occasionally stopped responding completely in particular components of the chain, because transitions out of the initial and middle links of the chain were response dependent, and in order to avoid the inclusion of long time periods in which no responding occurred, the response rates presented were calculated from only those components that were part of a completed chain.

As expected, response rates were graded across the three components of each chain, and there was no reli-
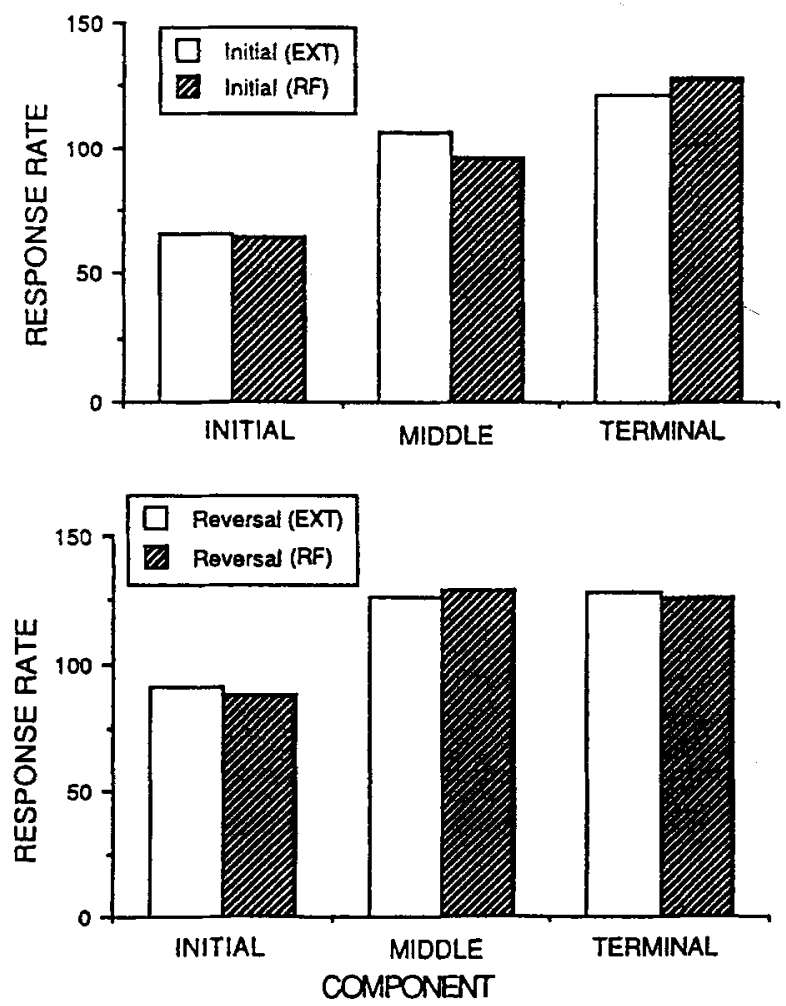

Figure 1. Mean responses per minute during the last five sessions of baseline training for each link of the two chain schedules, which are presented separately as a function of which chain had its terminal link subsequently extinguished (EXT) or reinforced (RF) during the devaluation phase. The top portion shows the results from the initial phase of training; the bottom portion shows the results from the reversal phase of training.
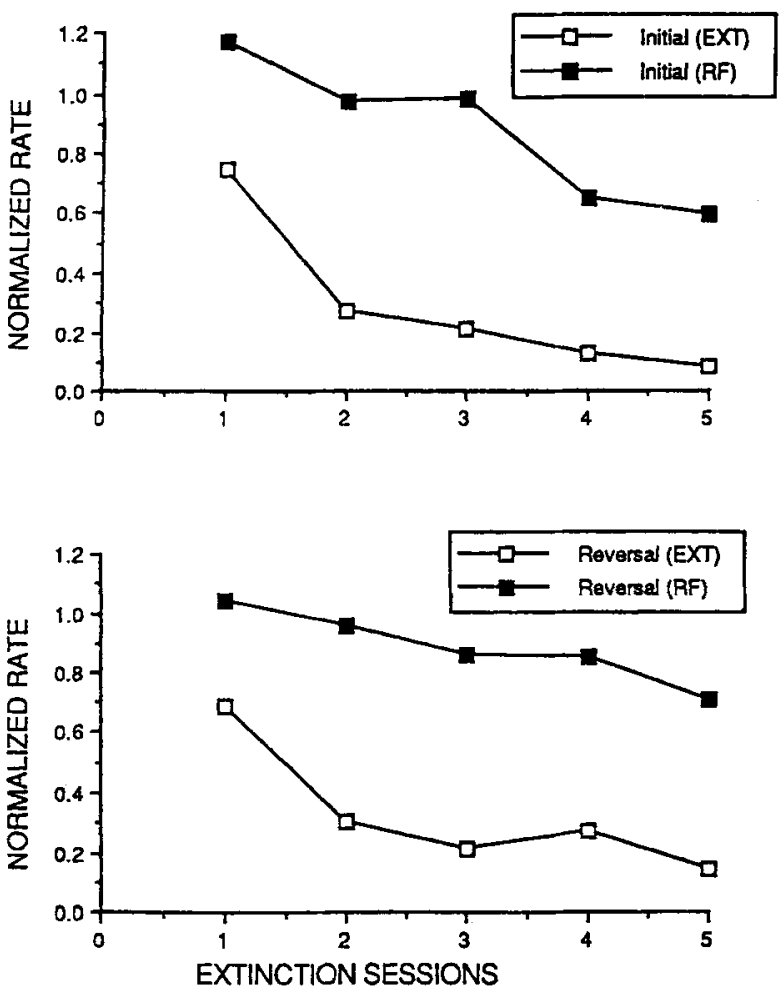

Figure 2. Response rates in the terminal link of each chain during the five sessions of extinction testing after devaluation training. Response rates were normalized for each subject relative to its response rate during the last five sessions of baseline training. Results in the top portion are from the initial phase of training; those in the bottom portion are from the reversal phase of training.

able difference between the components of the corresponding segments of the two chains. Response rates in the initial and middle links were slightly higher during the reversal phase of the experiment than during original training, making the gradient of response rate somewhat shallower for the reversal phase of the experiment as well.

The devaluation phase required a discrimination between the terminal-link stimuli of each chain, since one stimulus continued to be associated with the VI $30-\mathrm{sec}$ schedule and the other stimulus was associated with extinction. During the last session of this discrimination training, the discrimination index (responses to the VI stimulus divided by total responses) was .88 during the initial phase and .80 during the reversal phase. Thus, the discrimination training was successful in establishing differential behavior to the terminal-link stimuli of the two chains.

Because the stimulus context in which the discrimination training was administered was different from that in original training, it is important to establish that the discrimination training transferred to the terminal-link stimuli when the subjects were returned to the chain schedule. Figure 2 shows the response rates during the terminal links of the two chains across the five sessions of extinction testing when the entire three-link stimulus 
sequence again occurred. Response rates were normalized with respect to the response rates during the last five sessions of baseline training for each subject. It is evident that the discrimination training produced a difference in response rate on the first session of extinction testing, and that this difference increased over testing. Response rates decreased during both components but at a faster rate for the terminal-link stimulus correlated with EXT during discrimination training. The pattern was essentially similar for both the initial and the reversal phases of the study.

The pattern of results seen in Figure 2 was assessed statistically by a mixed analysis of variance (ANOVA), with group assignment as a between-subject variable and reinforcement history, phase of training (original vs. reversal), and session of extinction as within-subject variables. The only significant effects $(p<.05)$ were reinforcement history $[F(1,9)=194.6]$, sessions of extinction $[F(4,36)=36.9]$, and the interaction between reinforcement history and sessions of extinction $[F(4,36)=3.42]$. Analysis of simple effects showed that the effect of reinforcement history was significant for each individual extinction session, including the first session of testing immediately after separate discrimination training $[F(1,9)=27.1]$.

The results of major interest are the rates of extinction in the initial and middle links of the two chains, as a function of which terminal-link stimulus was correlated with EXT during the preceding devaluation training. Figure 3 shows these results subdivided into the two phases of training. The pattern of results was generally similar during both phases of training, but more pronounced during the reversal phase of the study. Normalized response rates were higher during the middle link than during the initial link, while the effect of the prior discrimination training appeared to be different as a function of the link of the chain. For the initial link, there was no consistent difference as a function of which terminal-link stimulus was devalued (initial EXT vs. initial $R F$ ), for any session during either phase of training. For the middle link, on the other hand, responses were generally lower for the chain with the devalued terminallink stimulus.

For the purposes of statistical analysis, the results from the two phases of training were averaged for individual subjects, in order to cancel any stimulus preferences, and then subjected to a mixed ANOVA in which group was a between-subject factor and reinforcement history, chain component (initial vs. middle links), and extinction session were within-subject factors. The main effect of groups was not significant $(F<1)$; the main effect of reinforcement history approached, but did not achieve, significance $[F(1,9)=4.57, .10>p>.05]$; the main effect of chain component was significant $[F(1,9)=21.8]$. The interaction between reinforcement history and chain component was significant $[F(1,9)=$ 6.54]. An analysis of simple effects showed that the effect of reinforcement history for the initial link was not significant $(F<1)$, whereas the effect of reinforcement
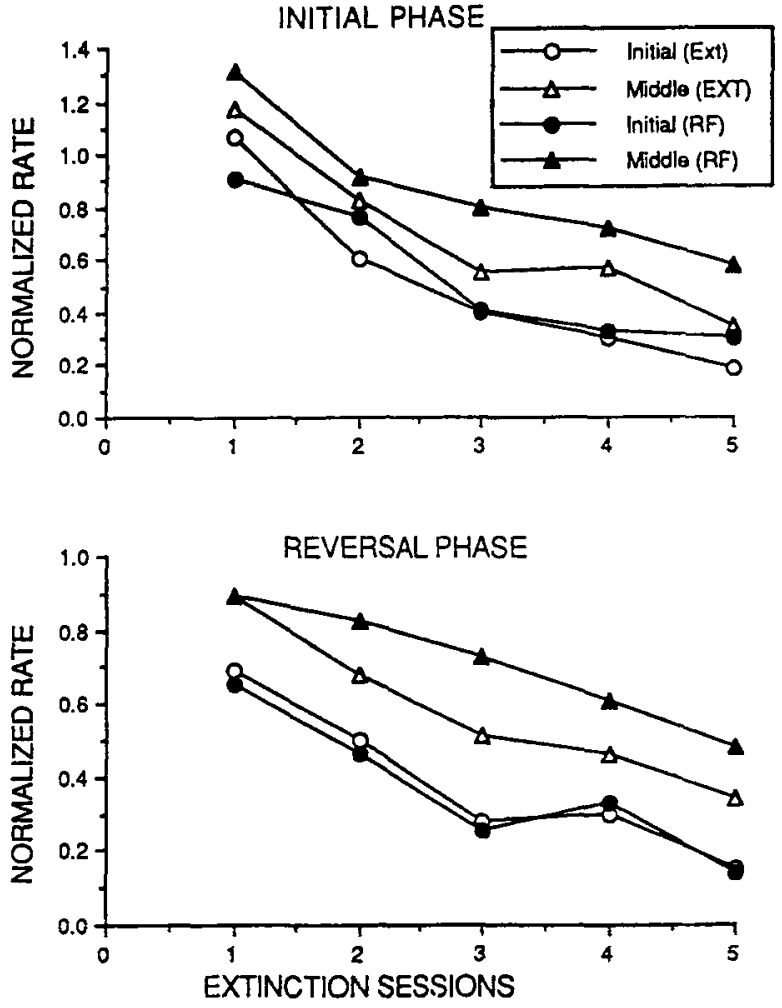

Figure 3. Normalized response rates in the initial and middle components of each chain during the five sessions of extinction testing after devaluation training. Results are the averages of all subjects.

history for the middle link was significant $[F(1,9)=$ 6.65]. Also, the effect of component was significant for both the chain with the terminal link devalued $[F(1,9)=$ $29.6]$ and the chain with the terminal link not devalued $[F(1,9)=7.39]$. The interaction between reinforcement history and extinction session was also significant $[F(4,36)=2.74]$, which reflects the faster rate of extinction for the chain with the terminal-link stimulus that was devalued. All other effects were nonsignificant, except for a significant triple interaction between group, component, and extinction session $[F(8,36)=2.78]$. This interaction involving the group variable was presumably due to differential stimulus generalization between the different components of the chain resulting from the different stimulus assignments for the different groups (see Procedure).

Because the major comparison of interest was whether the effects of devaluing the terminal-link stimulus of one of the chains had an immediate impact on responding at the start of the extinction phase, the results during the first session of extinction testing were examined more closely. To evaluate the pattern of extinction during the middle links, a separate ANOVA was conducted on the data from the first session alone, with group as a between-subject factor and reinforcement history as a within-subject factor. The effects of group $[F(2,9)=$ 1.20], the reinforcement history $(F<1)$, and their inter- 
action $[F(2,9)=1.12]$ were not significant. Restriction of the analysis to the first half of the first extinction session produced a similar result, with no evidence of any effect of devaluing the terminal link on middle-link performance.

A similar analysis was performed on the second session of extinction testing. Here the effect of group was also not significant $[F(2,9)=1.22]$, but the effect of reinforcement history was $[F(1,9)=6.24]$. The interaction was not significant $[F(2,9)=1.80]$. Thus, the effect of devaluing one of the terminal-link stimuli was evident in the middle link of the chain by the second session of extinction testing, but not during the first session.

\section{Discussion}

The pattern of results, while relatively clear, is not easily explained by any one simple principle. There was no differential effect of devaluing one terminal link on responding in the initial links of the chain, and also no effect on responding in the middle link of the chain on the first day of testing, but an effect on the latter did develop with continued testing. These results suggest that there was no direct transmission of the changed value of the terminal-link stimulus to the preceding links of the chain, but that such value transmission depended on continued pairings of middle-link responding with the terminal-link stimulus over the course of extinction testing.

The necessity of continued pairings of the middle and terminal links of the chain for showing any differential effects of terminal-link value is partially consistent with the traditional view of chain-schedule performance. Assuming that the effective reinforcement contingency for maintaining middle-link behavior was access to the terminal-link stimulus, the critical manipulation to produce extinction presumably is the occurrence of middle-link responding followed by the devalued terminal-link stimulus. This prediction can be questioned, however, on the basis of the results of the analogous second-order conditioning experiments noted in the introduction: Several different studies using the autoshaping preparation (e.g., Rashotte et al., 1977) have shown that extinction of S1 after second-order conditioning of S2 by $\mathrm{S} 2-\mathrm{S} 1$ pairings results in the reduction of responding to $S 2$ without needing further S2-S1 pairings after S1 devaluation. Given that both conditioning preparations involved pigeon's keypecking, it is not obvious why the outcome of the present study diverges from that of the earlier findings.

A potential factor in the differences between the devaluation effects of the present chain procedure and those of the previous autoshaping studies lies in the nature of the associative relationship that underlies behavior in the two situations. In the second-order conditioning of autoshaping, the associative relationship was presumably between two stimuli, S2 and S1. In a chain schedule, in contrast, an operant contingency is involved, so that the associative relationship was presum- ably between responding in the middle link and onset of the terminal-link stimulus, a response-stimulus relationship. The reason this difference may be important is that devaluation effects in the autoshaping procedure apparently depend upon stimulus similarity between S2 and S1. Nairne and Rescorla (1981) reported that when both S2 and S1 were keylights, extinction of S1 resulted in immediate decrements of responding to $S 2$, but when $\mathrm{S} 2$ was a keylight and S1 was an auditory cue, there was no immediate effect of $\mathrm{S} 1$ extinction on $\mathrm{S} 2$ responding. Given that the devaluation effect occurs only when the two events being associated are physically similar, it should not be surprising that it did not occur here, because the physical characteristics of the act of pecking and the onset of an illuminated key are quite different. Viewed from this perspective, the present devaluation effects are consistent with the view that the effective controlling relationship in chain schedules is between responding in one link of the chain and the conditioned reinforcement properties of the stimulus associated with the succeeding link.

Although the absence of an immediate effect of terminal-link devaluation can be viewed as consistent with the traditional conditioned reinforcement interpretation of chain schedule performance, the differential rates of extinction for the initial versus middle links are inconsistent with that view. For both chain components, regardless of the treatment of their respective terminallink stimuli, responding decreased more in the initial link of the chain than during the middle link. In contrast, a backward pattern of extinction would imply that extinction effects should have occurred more quickly during the middle link. The puzzle is how to reconcile the failure to obtain the backward pattern of extinction with the effects of the devaluation procedure, which were stronger during the middle link of the chain.

\section{EXPERIMENT 2}

One possible reason for the more rapid extinction during the initial than during the middle link of the chains in Experiment 1 is that the initial link was more strongly affected by the generalization decrement caused by the removal of reinforcement from the situation. During training, the delivery of reinforcement always preceded the onset of the initial link, whereas the onset of the middle link followed the initial link. Thus, when food was omitted at the onset of extinction, the stimuli prior to initial-link responding were changed substantially more than those for middle-link responding. For two of the three groups in Experiment 1, a 30-sec ITI was inserted between the end of the preceding chain and the onset of the initial link in order to reduce the impact of this difference, although it may still have played a significant role given that food delivery is a highly memorable cue. To avoid this problem in Experiment 2 the ITI between the end of one chain and the onset of the next was continued but now included a signaled food presentation 
midway through the ITI during both training and extinction testing. It was also our expectation that the continuation of food during the extinction test phase, while not contingent on chain-schedule performance, would substantially retard the rate of extinction, because the continued presence of food would maintain the incentive value of the conditioning situation.

A second change in the procedure for Experiment 2 was that the extinction test phase following devaluation training excluded the presentation of the terminal links. This modification is important in order to interpret the effect of the devaluation procedure on middle-link responding seen in Experiment 1 . It is possible that the failure to see an effect at the onset of the test phase was due simply to the first extinction session's not being a sufficiently sensitive test, perhaps because of the substantial reinstatement of responding during the extinguished terminal link itself, as seen in Figure 2. Thus, only after differences in responding in the two terminal links themselves became substantially larger would differences in the preceding links be likely to show differences as well. This possibility suggests that continued pairings of middle-link responding with terminal-link stimuli may not have been necessary for the differential performance in the middle links to develop. If so, a similar difference in the middle links of the two chains should occur even when the terminal links were omitted during extinction testing. On the other hand, if the development of differences in middle-link responding over the course of the extinction training did depend on the continued pairing of the middle-link stimuli with the terminal-link stimuli of different value, no difference in middle-link responding should be observed here.

A final addition to the procedure of Experiment 2 was the use of two different schedule values in the components of the chain schedule. Whereas VI $30-\mathrm{sec}$ schedules in all components of the schedule were used in Experiment 1 , here two separate groups were compared, one with VI 10-sec (short) components and the other with VI 60-sec (long) components. This comparison was included because it seemed plausible that the dynamics of extinction might depend critically on the absolute temporal distance between the initial and middle links of the chain to food at the end of the chain.

\section{Method}

\section{Subjects and Apparatus}

Eight new subjects, with histories similar to those used in Experiment 1, were maintained in the manner described for Experiment 1 . The same set of conditioning chambers was also used.

\section{Procedure}

The pretraining procedure was the same as that used in Experiment 1 . All subjects then received the two separate three-link chain schedules on a randomly alternating basis. For one group of 4 subjects, the schedule value for each link of both chains was a VI 60 -sec schedule. For the second group, the schedule value for each link was VI $10 \mathrm{sec}$. Response requirements for each component of the two chains always occurred on the left response key.
After the response-contingent food presentation at the completion of each chain schedule, a 30-sec ITI began, halfway through which the right response key was illuminated with a red vertical line, and a single peck to the right key delivered food and turned off the key for the duration of the ITI. At the end of the ITI, one or the other initial-link stimulus was presented on the left key and the next chain component was begun.

The stimuli associated with the different links of the two chains were partially counterbalanced across subjects. For 2 subjects within each group, the assignment of stimuli was red, green, and a horizontal white line for the initial, middle, and terminal links, respectively, of Chain 1 and white, blue, and a vertical white line for the corresponding components of Chain 2 . For the remaining 2 subjects, the assignments were green, red, and horizontal lines for the three successive components of Chain 1 and blue, white, and vertical line for the three components of Chain 2 . Thus, for all subjects, the different line orientations occurred in the terminal components of the two chains and diffuse colors occurred in the initial and middle links.

Training on the initial baseline condition continued for 25 sessions. Each session continued until 32 reinforcers had been earned at the end of the two chains, 16 from each chain schedule.

Following the initial baseline condition, devaluation sessions were conducted in which only the stimuli correlated with the two terminal links were presented (vertical and horizontal lines). These were alternated randomly with extinction in effect during one stimulus and the food reinforcement schedule used during the baseline condition (VI $60 \mathrm{sec}$ or VI $10 \mathrm{sec}$ ) in effect during the other. Which stimulus was correlated with reinforcement/extinction was counterbalanced across subjects. This devaluation training continued for 15 sessions, each consisting of 16 presentations of each stimulus. Throughout this training, the $30-\mathrm{sec}$ ITI continued to be presented between stimulus presentations, along with the signaled food presentation at the midpoint of the ITI.

Test sessions then began after completion of the devaluation phase. Here the two chains again alternated randomly, but now each chain terminated with the end of the middle-link stimulus without presentation of the terminal-link stimuli and, thus, without food. In this phase only, transitions between components were response-independent, occurring whenever the intervals timed by the VI clock had elapsed rather than after the first response following the interval. The ITI and signaled food delivery in the middle of the ITI continued to occur as before, however. Fifteen extinction test sessions were presented.

After completion of the three phases just described, the entire experiment was repeated exactly, except that the correlation between the terminal-link stimuli and the reinforcement contingencies was reversed during the devaluation phase.

\section{Results and Discussion}

There were no systematic differences between the original versus reversal phases of the experiment, so the results were averaged across the two phases for analysis and simplicity of presentation. Figure 4 shows the average response rates during the last five sessions of the baseline training phase, separately for the components that were to receive the different treatments with respect to the terminal-link stimuli during the devaluation phase. Shown in the top portion are the results for the subjects with VI 60 -sec schedules; in the bottom portion are the results for subjects with VI 10 -sec schedules. For both, the average response rate was lower during the initial component of the schedule than during the other two components, which were similar. With the VI 60-sec 

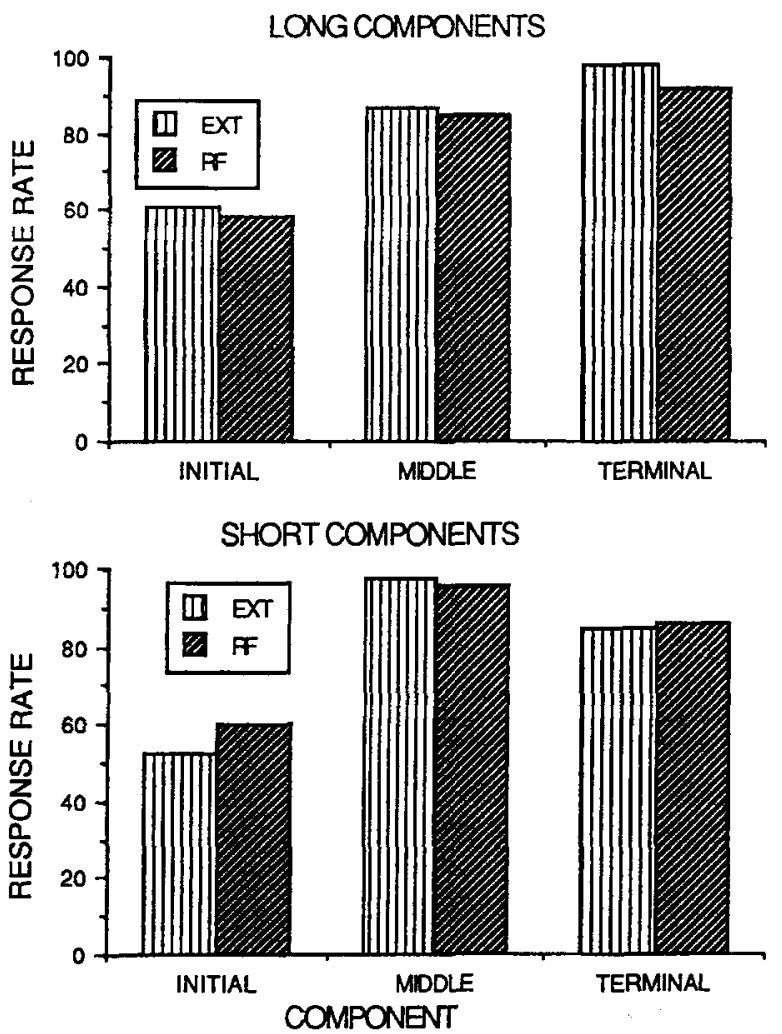

Figure 4. Mean responses per minute during the baseline phase of Experiment 2 for each component of the three-link chains subdivided according to the treatment with respect to the terminal-link stimulus during the devaluation phase. The top panel shows the data for subjects with VI 60-sec schedules in each component; the bottom panel shows the data for subjects with VI 10-sec schedules. Results were averaged over the initial and reversal phases of the procedure.

schedules, response rate was slightly higher in the terminal component than in the middle component, an effect that occurred for all 4 subjects. The opposite pattern occurred for the VI 10-sec schedules but was not consistent across subjects.

For all subjects, substantial discrimination between the two terminal-link stimuli developed during the 15 sessions of training with the devaluation phase: the average discrimination accuracy (proportion of total responses to the terminal-link stimulus associated with reinforcement) during the last five sessions of this training was .80 for the VI $60-\mathrm{sec}$ subjects and .85 for the VI 10 -sec subjects.

Figure 5 shows the results for subjects with the VI 60 -sec schedules over blocks of three successive test sessions. Larger units were needed because the overall rate of extinction was considerably slower than that seen in Experiment 1, presumably because of the continued presence of food in the ITI. Also, absolute response rates are plotted here instead of normalized rates, because stimulus preferences across the components were less evident and because preliminary analysis indicated that the pattern of results was entirely similar for the normalized rate measure. The four functions seen in Fig- ure 5 correspond to the initial and middle links of the chains, subdivided according to whether the terminallink stimuli that followed them during the baseline phase of training were later associated with reinforcement (RF) or extinction (EXT). Here, unlike Experiment 1, extinction was slower during the initial component of the schedule than during the middle component; also, there is little evidence of a drop in responding across the 15 extinction sessions for initial-link responding, although there is a substantial decrease in middle-link responding. For neither component was there a consistent difference as a function of which chain had its terminallink stimulus correlated with extinction; the small difference evident for the middle-link responding was not reliable and was in the opposite direction of what would be expected on the basis of the extinction manipulation.

The results shown in Figure 5 were subjected to a within-subject ANOVA, with the factors of component (initial vs. middle links of the chain), reinforcement history during the devaluation phase (RF vs. EXT), and blocks of test sessions. The main effect of component was significant $[F(1,3)=10.06]$, the effect of reinforcement history was not significant $(F<1)$, and the main effect of test sessions was significant $[F(4,12)=6.09]$. The interaction between component and test sessions was also significant $[F(4,12)=10.39]$, and an analysis of simple effects revealed that the effect of test sessions was significant for the middle link, but not for the initial link $(F<1)$. Also, the effect of component was not significant for the first two blocks of three test sessions, but was significant for each of the remaining three blocks. The interactions between reinforcement history and component and between reinforcement history and test sessions were not significant (both $F$ s $<1$ ). However, the triple interaction was significant $[F(4,12)=$ 3.42], although the basis of the interaction is unclear from Figure 5.

The results during the test sessions for the VI 10-sec schedules are shown in Figure 6. Here the response rates

\section{60-SEC COMPONENTS}

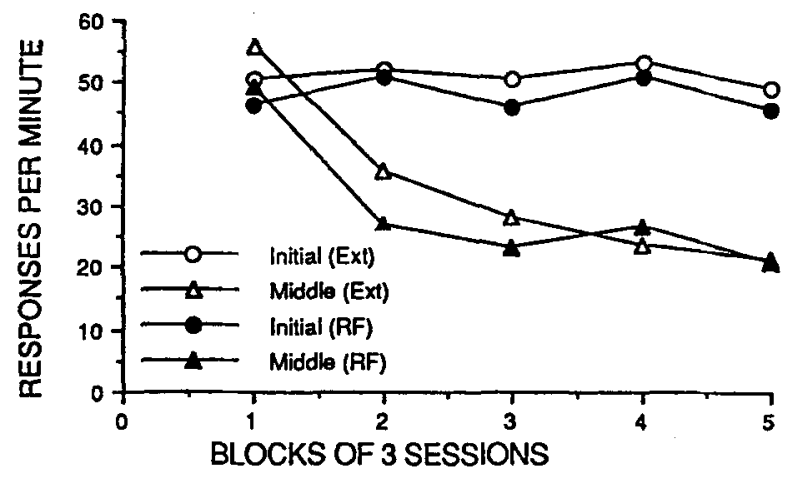

Figure 5. Results from the extinction phase of Experiment 2 for subjects trained with VI 60 -sec schedules in each link of the chain. Response rates are subdivided according to the link of the chain, and as a function of which chain had its terminal-link stimulus extinguished or reinforced during the devaluation phase. 


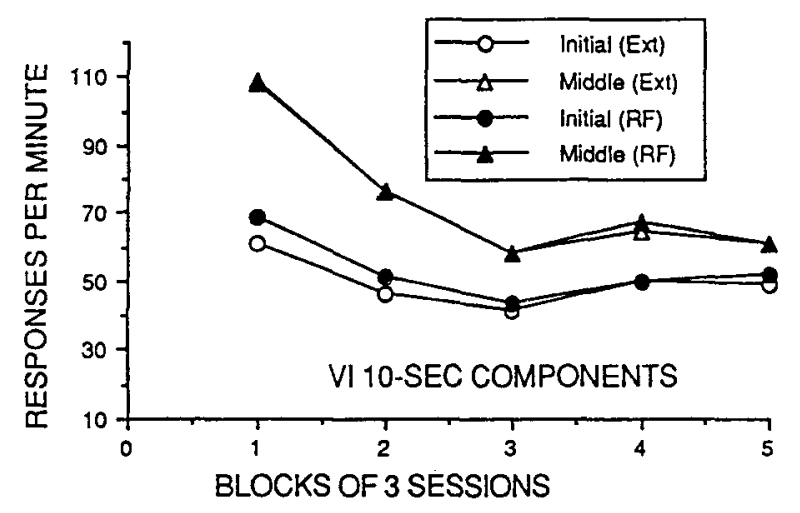

Figure 6. Results from the extinction phase of Experiment 2 for subjects trained with VI 10-sec schedules. Response rates are subdivided according to the link of the chain, and as a function of which chain had its terminal-link stimulus extinguished or reinforced during the devaluation phase.

were higher during the middle component of the schedule than during the initial component throughout extinction testing, although response rates in the middle component of the schedule also declined at a more rapid rate. Unlike in Figure 5, some evidence of a decline is also evident for initial-link responding as well. For neither component is there any evidence of a significant effect of which chain schedule was devalued prior to the test.

The results of the three-way ANOVA for the VI 10-sec schedule were that neither the main effect of extinction versus reinforcement during the devaluation procedure nor the main effect of component was significant $[F(1,3)=3.35$ and $F<1$, respecitvely]. The interaction between reinforcement history and component was also not significant $[F(1,3)=1.05]$. The main effect of extinction sessions was significant $[F(4,12)=5.78]$, as was the interaction between component and extinction sessions $[F(4,12)=4.94]$. Analysis of simple effects revealed that the components did not differ reliably at any of the five blocks; however, the effects of sessions was significant for the middle link $[F(4,12)=9.26]$ although not for the initial link $(F=1.62)$. Thus, the significant interaction was due to the greater reduction in response rate that occurred during the middle link of the schedule.

\section{Discussion}

The results of Experiment 2 differed in two significant ways from those of Experiment 1. First, the rate of extinction for both schedule values was greater during the middle link of the schedule than during the initial link, in contrast to the opposite pattern seen in Experiment 1 . The most likely source of this reversal in pattern is the elimination in Experiment 2 of the confound present in Experiment 1 of a greater generalization decrement for the initial link of the chain caused by the removal of food from the situation. Here the role of food cues before the onset of the initial links remained constant because of the maintenance of food delivery during the ITI both during training and extinction testing. The result was that no significant reduction in responding occurred during the initial links across all 15 extinction sessions for either schedule value studied. The implication is that responding in the initial links of a chain is substantially resistant to extinction, given that food continues to be present in the situation. How much such resistance to extinction depends on the continued incentive properties of the food versus the constancy of the stimulus conditions at the onset of initial-link responding remains an open question.

The second factor that may have contributed to the faster rate of extinction during the middle links of the schedule was the removal of the reinforcer supporting middle-link responding. In the extinction sessions of Experiment 1 , middle-link responding produced the onset of the terminal-link stimulus, whereas in Experiment 2 such responding had no effect and was followed by the onset of the ITI. The result was that middle-link responding declined to a low level during Experiment 2, although the absolute rates of behavior cannot be directly compared because of the generally faster rates of extinction in Experiment 1. Nevertheless, comparison of middle-link responding across the two experiments suggests that the conditioned reinforcement value of the onset of the terminal-link stimulus was a significant determinant of middle-link response rate.

The second major difference between the results of the two experiments is that Experiment 2 failed to find any effect of the terminal-link devaluation procedure on either initial or middle-link responding. The failure to find an effect on initial-link responding is consistent with the findings of Experiment 1, but the absence of an effect for the middle link stands in contrast to the finding of Experiment 1. This difference in the pattern of middlelink responding implies that the lower response rate in the middle link of the chain followed by the devalued terminal link in Experiment 1 was due to the continued pairings of the middle and terminal links, and thus was due to the pigeon's learning that responding during one of the middle links was followed by a less valuable stimulus. In the absence of those pairings, we found, in Experiment 2 , no difference between the two chains as a function of which terminal-link stimulus was devalued.

\section{GENERAL DISCUSSION}

The different patterns of extinction for the initial and middle links of the chain schedules obtained in Experiment 1 versus Experiment 2 may help to elucidate the basis of the conflicts in the existing literature. As noted in the introduction, Fantino (1965, Experiment 1) observed a faster rate of extinction in the terminal link of a two-link chain than in the initial link. A similar backward pattern of extinction was reported by Rescorla (1977), who trained rats in a complex maze and then al- 
lowed them to run under conditions of food satiation. During initial testing, there was little effect of food satiation, but then the pattern of deterioration was that the maze segments nearest the goal were most affected, while those farthest from the goal were least affected. This backward pattern of disruption is inconsistent with the analysis of chain-schedule performance in terms of the notion of "resistance to change" by Nevin et al. (1981), who reported that response rate in the initial link of a chain was more easily reduced by motivational variables (decreased food motivation, additional reinforcement in the situation) than was response rate in the terminal link of the chain. Their analysis implies that response strength was less in the initial link than in the terminal link, which suggests that any measure of response strength, including resistance to extinction, should reflect such response-strength differences.

Because we produced both patterns of extinction in the present two experiments, a comparison of their procedural differences should be informative. In Experiment 2, behavior in the middle link extinguished rapidly, while that during the initial link changed very little across the entire 15 sessions of training. In contrast, the extinction sessions of Experiment 1 produced a greater disruption of responding in the initial link of the chain. The most obvious difference between the two procedures was the continued presence of food in the situation in Experiment 2 despite the extinction contingencies for chain performance itself. It is likely that the occurrence of food was a substantial part of the stimulus complex controlling responding in the initial link of the schedule, so that its removal during the extinction phase of Experiment 1 had the effect of producing a greater performance decrement in the initial link than the middle link, where the occurrence of food was presumably a much smaller portion of the stimulus complex controlling responding. A similar explanation might be applicable for the resistance-to-change manipulations of Nevin et al. (1981), in that food satiation should presumably change the stimulus character of the food, just as does removing the food completely. Thus, because of the greater generalization decrement, response rate should be more disrupted in the initial link of the chain than in the later links.

Taken together, the present two experiments offer strong support for the traditional interpretation of chainschedule performance, which assumes that behavior in each link of the chain is maintained by the conditioned reinforcement value of the stimulus associated with the succeeding link of the chain. The finding in Experiment 1 , that separate extinction training during the terminal link of one chain increased the rate of extinction during the preceding middle link when the entire chain was extinguished together, strongly supports the interpretation that middle-link responding was maintained by the conditioned reinforcement value of the terminallink stimulus. Similarly, the backward pattern of extinction seen in Experiment 2 is expected from the assumption that the conditioned reinforcement value of the middlelink stimulus must be lost before extinction of initiallink responding occurs. Neither of these findings can be explained by the alternative interpretation of chainschedule performance offered by Staddon (1983), who argues that response rates in the various links of a chain are the result of the temporal distances to reinforcement signaled by each stimulus composing the chain sequence. By this account, there is no obvious reason why extinguishing the terminal-link stimulus of one chain should differentially affect responding of the preceding links of the same chain. Similarly, there is no basis for expecting the backward pattern of extinction obtained in Experiment 2 . The failure of the "cued temporal distance" hypothesis to explain these features of the data does not mean, of course, that the discriminative properties of the various stimuli do not contribute to the type of behavior pattern that is obtained. At present, however, there is no clear evidence that these discriminative properties are in any strong way related to the time to primary reinforcer. In contrast, several lines of evidence suggest that time to the next link in the chain does control behavior (e.g., Royalty, Williams, \& Fantino, 1987; Williams \& Royalty, 1990). Thus, the concept of conditioned reinforcement, rather than the molar notion of "time to primary reinforcement," offers the most appropriate theoretical framework for understanding chainschedule performance.

Finally, some comment is warranted regarding the complete insensitivity of responding in Experiment 2 (and of initial-link responding in Experiment 1) to the devaluation of one of the terminal-link stimuli outside of the chain procedure. As noted in the introduction, analogous studies of autoshaping have found that responding elicited by a stimulus conditioned via second-order conditioning is diminished when the first-order stimulus is extinguished (e.g., Rashotte et al., 1977). However, similar procedures using other types of responses (e.g., conditioned activity) have not been found to be sensitive to devaluation of the first-order stimulus (e.g., Holland \& Rescorla, 1975). It is noteworthy that the results of the present study are consistent with the only other study of chain schedules using the devaluation procedure that we have been able to find. Moore (1986) trained pigeons on a three-link chain schedule with brief (3-sec) components. After initial training, in which the three-link chain was followed by food, the middle- and terminal-link components of the schedule were presented together followed by no food. Then, during a test phase without further reinforcement of the chain, response rate to the initial-link stimulus was maintained at a high rate, although no control condition was included to allow a sensitive index of whether there was any diminution of responding due to the devaluation of the later links of the chain. It thus appears that, at least with the type of chain used here, modifications of the value of an element of a chain outside the context of the chain will have no immediate effect on performance in the earlier links of the 
chain. This finding is important because it suggests that chain-schedule performance may be very different from the simple instrumental conditioning situations in which sensitivity to changing the value of the contingent reinforcer has been consistently demonstrated (e.g., Colwill $\&$ Rescorla, 1986). The further implication is that instrumental behavior may or may not be sensitive to changes in the value of the reward contingent on the behavior, depending on the extent to which behavior is controlled directly by the primary reward or by stimuli intervening between the response and the reward. In most instrumental learning situations, substantial stimulus change precedes the presentation of the primary reinforcer (e.g., the illumination and sound of food-hopper activation), and it is possible that the behavior is substantially under the control of these stimuli (cf. Zimmerman, Hanford, \& Brown, 1967). Given such control by stimuli intervening between the operant response and primary reinforcer, it seems plausible that operant behavior trained even with simple reinforcement contingencies might be impervious to modifications of the current value of the primary reward, as with the chainschedules studied here.

\section{REFERENCES}

AdAms, C. D., \& Dickinson, A. (1981). Actions and habits: Variations in associative representations during instrumental learning. In N. E. Spear \& R. R. Miller (Eds.), Information processing in animals: Memory mechanisms (pp. 143-165). Hillsdale, NJ: Erlbaum.

Colwill, R. M., \& Rescorla, R. A. (1986). Associative structures in instrumental learning. In G. H. Bower (Ed.), The psychology of learning and motivation (Vol. 20, pp. 55-104). San Diego: Academic Press.

Dickinson, A. (1980). Contemporary animal learning theory. Cambridge: Cambridge University Press.

FANTINO, E. (1965). Some data on the discriminative stimulus hypothesis of secondary reinforcement. Psychological Record, 15, 409-415.

GolluB, L. (1977). Conditioned reinforcement: Schedule effects. In W. K. Honig \& J. E. R. Staddon (Eds.), Handbook of operant behavior (pp. 288-312). Englewood Cliffs, NJ: Prentice-Hall.
Holland, P. C., \& Rescorla, R. A. (1975). The effect of two ways of devaluing the unconditioned stimulus after first- and second-order appetitive conditioning. Journal of Experimental Psychology: Animal Behavior Processes, 1, 355-363.

Keller, F. S., \& Schoenfeld, W. N. (1950). Principles of psychology. New York: Appleton-Century-Crofts.

Moore, J. (1986). On the consequences of conditioning. Psychological Record, 36, 39-61.

Nairne, J. S., \& Rescorla, R. A. (1981). Second-order conditioning with diffuse auditory reinforcers in the pigeon. Learning \& Motivation, 12, 65-91.

Nevin, 3. A., Mandell., C., \& Yarensky, P. (1981). Response rate and resistance to change in chained schedules. Journal of Experimental Psychology: Animal Behavior Processes, 7, 278-294.

Rashotte, M. E., Griffin, R. W., \& Sisk, C. L. (1977). Second-order conditioning of the pigeon's key peck. Animal Learning \& Behavior, 5, 25-38.

Rashotte, M. E., Marshall, B. S., \& O'ConNell, J. M. (1981). Signaling functions of the second-order CS: Partial reinforcement during second-order conditioning of the pigeon's keypeck. Animal Learning \& Behavior, 9, 253-260.

ResCORLA, R. A. (1977). Pavlovian second-order conditioning: Some implications for instrumental behavior. In H. Davis \& H. M. B. Hurwitz (Eds.), Operant-Pavlovian interactions (pp. 133-164). Hillsdale, NJ: Erlbaum.

RESCORLA, R. A. (1979). Aspects of the reinforcer learned in secondorder Pavlovian conditioning. Journal of Experimental Psychology: Animal Behavior Processes, 5, 79-95.

Royalty, P., Williams, B. A., \& Fantino, E. (1987). Effects of delayed conditioned reinforcement in chain schedules. Journal of the Experimental Analysis of Behavior, 47, 41-56.

SKINNER, B. F. (1938). The behavior of organisms. New York: AppletonCentury-Crofts.

Staddon, J. E. R. (1983). Adaptive learning and behavior, Cambridge: Cambridge University Press.

Williams, B. A., \& RoYalty, P. (1990). Conditioned reinforcement versus time to reinforcement in chain schedules. Journal of the Experimental Analysis of Behavior, 53, 381-393.

Zimmerman, J., HANFord, P. V., \& Brown, W. (1967). Effects of conditioned reinforcement frequency in an intermittent free-feeding situation. Journal of the Experimental Analysis of Behavior, 10, 331 340.

(Manuscript received November 18, 1992; revision accepted for publication May 17, 1994.) 